\title{
H.761 Support of a New <concept> Element and a New "recognition" Node-Event to Enable Deep Learning-based Analyses for Media-Nodes
}

\author{
Antonio Busson \\ TeleMídia - PUC-Rio \\ busson@telemidia.puc-rio.br
}

\author{
Alan L. V. Guedes \\ TeleMídia - PUC-Rio \\ alan@telemidia.puc-rio.br
}

\author{
Sergio Colcher \\ Informatics Departament - PUC-Rio \\ colcher@inf.puc-rio.br
}

\begin{abstract}
Machine Learning field, methods based on Deep Learning (e.g. CNN, RNN) becomes the state-of-the-art in several problems of the multimedia domain, especially in audio-visual tasks. Typically, the training of Deep Learning Methods is done in a supervised manner, and it is trained on datasets containing thousands/millions of media examples and several related concepts/classes. During training, the Deep Learning Methods learn a hierarchy of filters that are applied to input data to classify/recognize the media content. In computer vision scenario, for example, given image pixels, the series of layers of the network can learn to extract visual features from it, the shallow layers can extract lower-level features (e.g. edges, corner, contours), while the deeper combine these features to produce higher-level features (e.g. textures, part of objects). These representative features can be clustered into groups, each one representing a specific concept. H.761 NCL currently lacks support for Deep Learning Methods inside their application specification. Because those languages still focus on presentations tasks such as capture, streaming, and presentation. They do not consider programmers to describe the semantic understanding of the used media and handle recognition of such under-standing. In this proposal, we aim at extending NCL to provide such support. More precisely, our proposal able NCL application support: (1) describe learning-based on structured multimedia datasets; (2) recognize content semantics of the media elements in presentation time. To achieve such goals, we propose, an extension that includes: (a) the new <knowledge> element describe concepts based on multimedia datasets; (b) <area> anchor with an associated "recognition" event that describes when a concept occurrences in multimedia content.
\end{abstract}

\section{KEYWORDS}

NCL, Ginga

\section{BACKGROUND}

Machine Learning field, methods based on Deep Learning (e.g. CNN, RNN) becomes the state-of-the-art in several problems of the multimedia domain, especially in audio-visual tasks. Typically, the training of Deep Learning Methods is done in a supervised manner, and it is trained on datasets containing thousands/millions of media examples and several related concepts/classes. During training, the Deep Learning Methods learn a hierarchy of filters

In: Future of Interactive Television Workshop (V WTVDI), Rio de Janeiro, Brasil. Anais Estendidos do Simpósio Brasileiro de Sistemas Multimídia e Web (WebMedia). Porto Alegre: Sociedade Brasileira de Computação, 2019.

ISSN 2596-1683 that are applied to input data to classify/recognize the media content. In computer vision scenario, for example, given image pixels, the series of layers of the network can learn to extract visual features from it, the shallow layers can extract lower-level features (e.g. edges, corner, contours), while the deeper combine these features to produce higher-level features (e.g. textures, part of objects). These representative features can be clustered into groups, each one representing a specific concept.

H.761 NCL [2] currently lacks support for Deep Learning Methods inside their application specification. Because those languages still focus on presentations tasks such as capture, streaming, and presentation. They do not consider programmers to describe the semantic understanding of the used media and handle recognition of such under-standing.

\section{PROPOSAL}

- (1) describe learning-based concepts on structured multimedia datasets using the new <concept> element;

- (2) recognize content semantics of the media elements in presentation time using a virtual anchor, called SemanticAnchor, with an associated new "recognition" event.

The overview of the proposal is illustrated in the next Figure

The <concept> element aiming at group and associate media datasets. It consists of an NCM Composite Node. The Composite Node elements (e.g. <body $>$ and $<$ context $>$ ) are useful to define compositions of multimedia data. That way, we <concept $>$ to represent whole media datasets for specific concepts, as well to specify the associations between them. All <concept $>$ s elements in a document are grouped in the $<$ knowledged $>$ at $<$ head $>$. We define two types of associations among <concept $>$ s:

- The hierarchy association defines a parenthood relation where is applied the rule: $\left(c_{1} \Rightarrow c_{2}\right)$, the concept node $c 2$ has media features from concept node $\mathrm{c} 1$, where $\mathrm{c} 1$ and $\mathrm{c} 2$ are called parent and child concepts, respectively.

- The mereology association defines a parthood relation, where is applied the rule: $\left(c_{1} \vdash c_{2}\right)$, the concept node $c_{2}$ is part of concept node $c_{1}$, this association indicates that media from $c_{2}$ are parts of media of $c_{1}$.

The following < knowledge $>$ code illustrate the definition of the <concept $>$ s elements and their media sets. In particular, the "tony_face" and "jony_face" < concept>s have hierarchy association "face" <concept>, consequently, "person" <concept>. Moreover, "face_p" <port $>$ " define a mereology. In other words, "face" is a part of "person". 


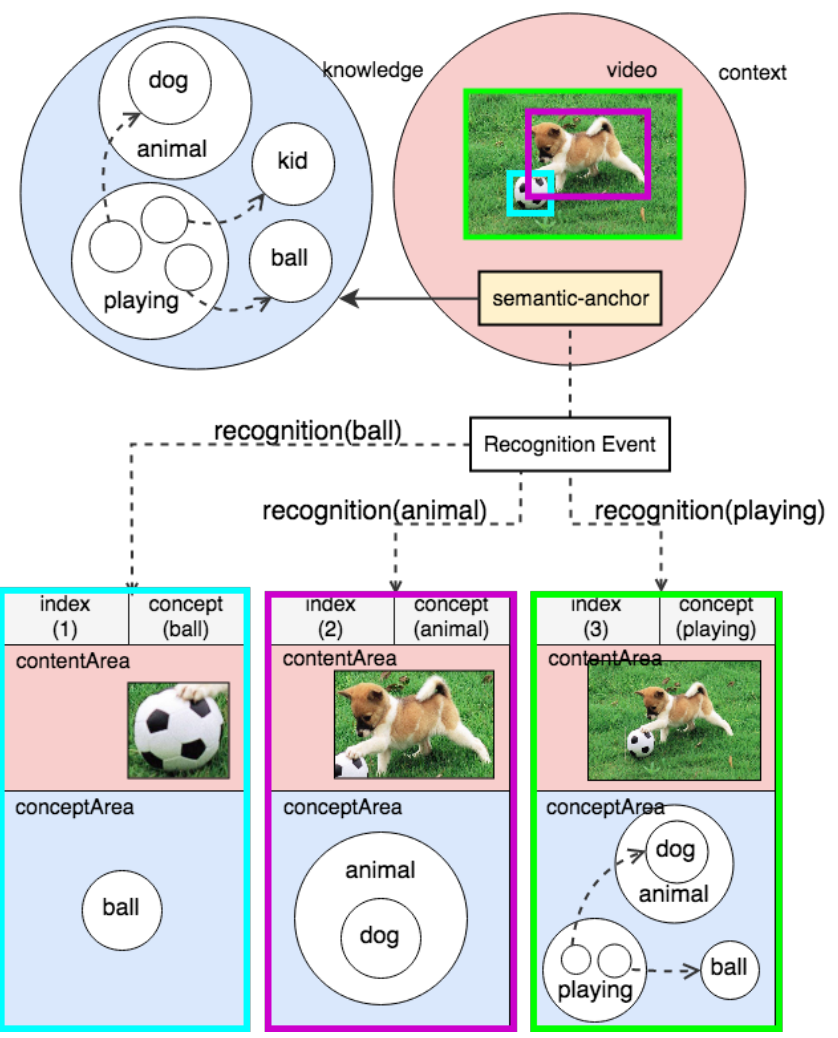

Figure 1: Example of three recognition events.

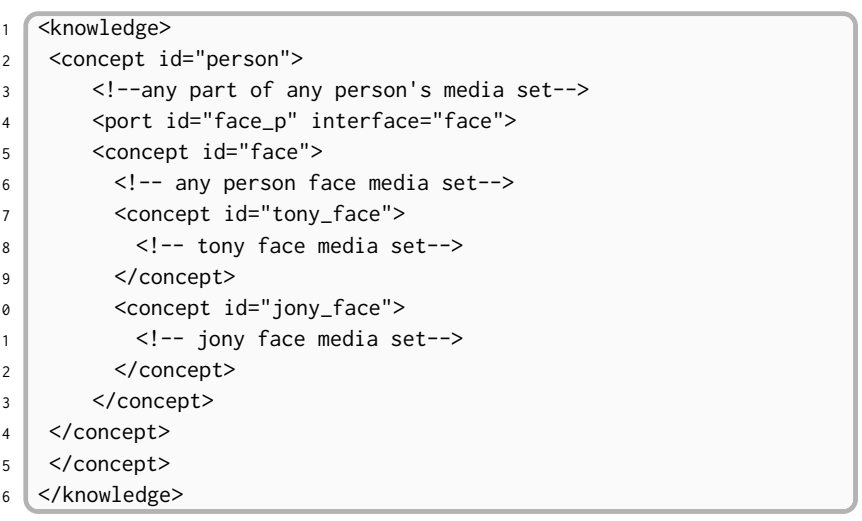

\section{Listing 1: NCL code for knowledge definition.}

Current NCL $<$ media $>$ may define bounding boxes through coords $<$ area $>$ attribute. Additionally, NCL $<$ media $>$ may define time intervals where concepts occurrences are detected through are begin $<$ area $>$ attribute. However, those < area $>$ usually are defined in the authoring phase and just occur in the execution phase. Our virtual < area> anchor with an associated "recognition" event aiming at recognizing content semantics of the media elements in presentation time. This proposal is based on the paper of Busson. al. "Embedding Deep Learning Models into Hypermedia Applications" (accepted to be published)[1].

\section{USE CASE}

The following code illustrate the usage of the <concept $>$ element. The $<$ head $>$ presents of $<$ concept $>$ s elements and their media sets. in particular, the "dog" <concept $>$ has hierarchy association with "animal" <concept>, whereas "head" <concept>" has mereology association with "dog" <concept>. The NCL link at lines 28-33 will be trigger when a dog appears in the video.

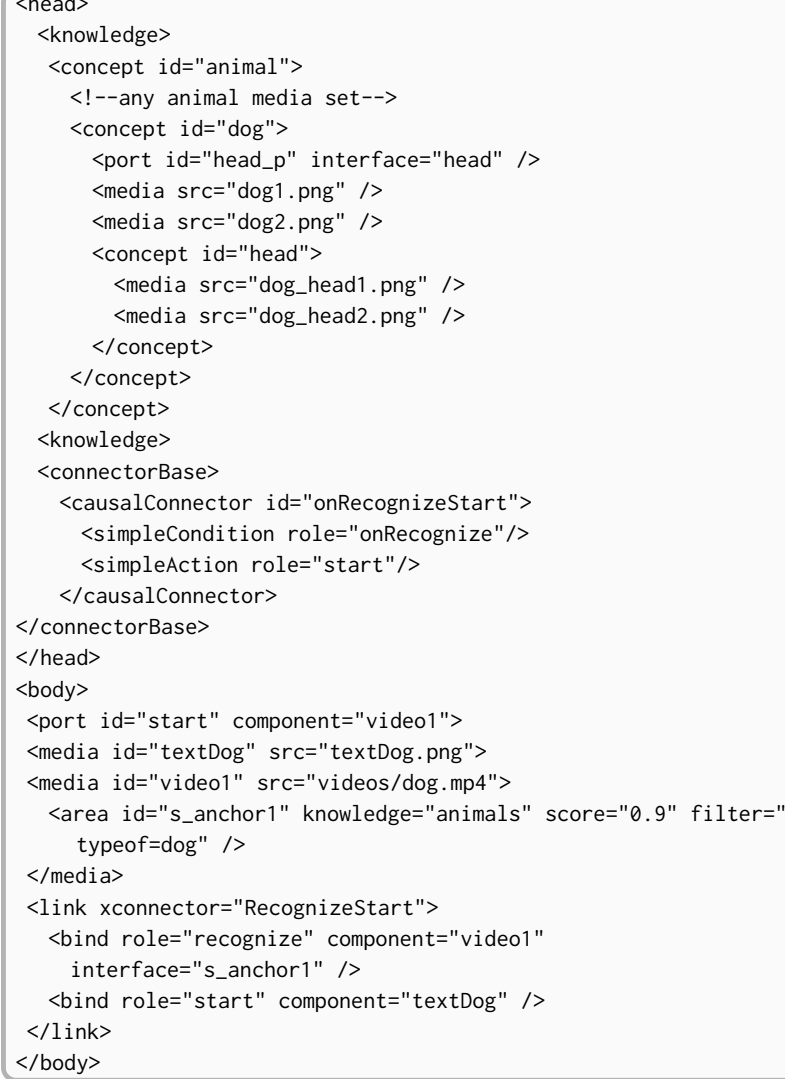

Listing 2: NCL code fragment using the proposed approach.

\section{REFERENCES}

[1] Antonio Busson, Álan Lívio Guedes, and Sergio Cocher. 2019. Embedding Deep Learning Models into Hypermedia Applications. In Lecture Notes in Computer Science (LNCS). (accepted to be published).

[2] ITU. 2009. H.761: Nested Context Language (NCL) and Ginga-NCL for IPTV Services. Technical Report. ITU, Geneva, Switzerland. https://www.itu.int/rec/T-REC-H. 761 